\title{
Simulations of Recoiling Massive Black Holes
}

\author{
Javiera Guedes ${ }^{1}$, Piero Madau ${ }^{1}$, Lucio Mayer ${ }^{2}$, Michael Kuhlen ${ }^{3}$, \\ Jürg Diemand ${ }^{2}$, and Marcel Zemp ${ }^{4}$ \\ ${ }^{1}$ Department of Astronomy \& Astrophysics, University of California, Santa Cruz, \\ 1156 High Street, Santa Cruz, CA 95062, USA \\ Email: javiera@ucolick.org \\ ${ }^{2}$ Institute for Theoretical Physics, University of Zürich, Winterthurerstrasse 190, CH-9057 \\ Zürich, Switzerland \\ ${ }^{3}$ Theoretical Astrophysics Center, University of California Berkeley 601 Campbell Hall, \\ Berkeley, CA 94720, USA \\ ${ }^{4}$ Department of Astronomy, University of Michigan, Ann Arbor, MI 48109, USA
}

\begin{abstract}
The coalescence of black hole binaries is a significant source of gravitational wave radiation. The typically asymmetric nature of this emission, which carries linear momentum, can result in the recoil of the black hole remnant with velocities in the range $100<V_{\text {recoil }}<$ $3750 \mathrm{~km} \mathrm{~s}^{-1}$. The detectability of recoiling massive black holes (MBH) as off-nuclear QSOs is tightly connected with the properties of the host galaxy, which determine the MBH's orbit and fuel reservoir. We present the results of $N$-body simulations of recoiling MBHs in high-resolution, non-axisymmetric potentials. We find that if the recoil velocities are high enough to reach regions of the galaxy dominated by the generally triaxial dark matter distribution, the return time is significantly extended when compared to a spherical distribution. We also perform simulations of recoiling MBHs traveling in gas merger remnants, where large amounts of gas have been funneled to the central regions, In this case, the MBHs remain within $R<1 \mathrm{kpc}$ from the center of the host even for high recoil velocities $\left(V_{\text {recoil }}=1200 \mathrm{~km} \mathrm{~s}^{-1}\right)$ due to the compactness of the remnant galaxy's nuclear disk. We discuss the implications of both scenarios for detectability.
\end{abstract}

Keywords. galaxies: nuclei, gravitational waves, methods: n-body simulations

Axisymmetry is typically broken in real galaxy mergers. To grasp the extent to which deviations from symmetry are important to the detectability of recoiling MBHs as offnuclear QSOs, we first study the effect of the triaxiality of the dark matter distribution using full $N$-body simulations of MBHs embedded in the main halo of the Via Lactea I simulation (Diemand et al. 2007). We use MBH orbits in pure dark matter simulations to constrain a model of a composite galaxy (a stellar spheroid plus a triaxial halo) and find that ejected MBHs with initial recoil velocities $V_{\text {recoil }}>500 \mathrm{kms}^{-1}$ do not return to the center of the host within a Hubble time (Guedes et al. 2008, 2009). To assess the effect of gas, we run simulations of recoiling MBHs in the high-resolution merger of Mayer et al. (2007). In this case, MBHs are not removed from the nuclear region. In both cases, detectability is challenging. In dry mergers, for instance, apocenter distances are high but the MBH fuel reservoir is scarce, while the opposite is true in major gas mergers.

\section{References}

Diemand, J., Kuhlen, M., \& Madau, P. 2007, ApJ, 657, 262

Guedes, J., Diemand, J., Zemp, M., Kuhlen, M., Madau, P., \& Mayer, L. 2008, AN, 329, 1004 Guedes, J., Madau, P., Kuhlen, M., Diemand, J., \& Zemp, M. 2009, ApJ, 702, 890

Mayer, L., Kazantzidis, S., Madau, P., Colpi, M., Quinn, T., \& Wadsley, J. 2007, Science, 316, 1874 